\title{
Air pollution and emergency department visits for urticaria
}

\begin{abstract}
Introduction. This study investigates associations between air pollution and emergency department (ED) visits for urticaria in Toronto, Canada.

Aim. To verify the hypothesis that urticaria are related to air pollution.

Material and methods. The National Ambulatory Care Reporting System database is used to draw the daily ED visits. The L50 section of the International Classification of Disease $10^{\text {th }}$ Revision is applied to extract ED visits whose primary causes was urticaria-related skin condition. Statistical models (condition Poisson regression) using daily counts of ED visits are constructed for urticaria (health response) with ambient air pollution concentrations and weather factors as independent variable. Two air quality health indexes and six ambient air pollutants: fine particulate matter $\mathrm{PM}_{2.5}, \mathrm{O}_{3}, \mathrm{CO}, \mathrm{NO}_{2}, \mathrm{SO}_{2}$, and maximum 8-hour average ozone are considered as an exposure.

Results. A total of 176 statistically significant $(\mathrm{P}-$ Value $<0.05)$ positive correlations were identified over the 15 day lag period (0-14 days). For daily average of ambient ozone, 74 positive correlations were observed with the following relative risks (RR) for a one interquartile range $(\mathrm{IQR}=12.8 \mathrm{ppb})$ increase: $\mathrm{RR}=1.361$ (95\% confidence interval: $1.302,1.404), 1.359(1.299,1.401)$, $1.351(1.281,1.404)$ in the warm season (April-September), lag 0, and RR=1.019 (1.013, 1.025), $1.023(1.016,1.030), 1.014$ (1.007, 1.021), lag 1, in the cold period (October-March), for all, females, and males, respectively. 10, 45 and 45 positive correlations were also obtained for sulfur dioxide, fine particulate matter, and daily maximum 8-hour average ozone concentrations, respectively.
\end{abstract}

Conclusions. The results indicate that urban ambient air pollution could influence the numbers of ED visits for urticaria. Ambient ozone was determined as the main environmental factor contributing to these associations.

Keywords: air pollution; concentration; counts; urban; sex; skin.

DOI: $10.2478 /$ pjph-2021-0013

\section{INTRODUCTION}

Hives (urticaria) are a common health condition characterized by red, itchy welts that result from a skin reaction. The lesions of urticaria are characterized by local transient skin and an area of redness [1]. The disease occurs with a sudden appearance of erythematous patches with pruritus [2]. The urticaria is heterogeneous among populations. Acute spontaneous urticaria episodes last for up to six weeks [3]. At least $15-20 \%$ of the global population will suffer from urticaria at some point in their lives. The burden of urticaria is high and may affect various aspects of the quality of life, including social isolation [4].

There is literature which reports the associations of urticaria with air pollution concentration levels. Identified among the ambient air pollutants related to urticaria were ozone [5], the air quality health index (encapsulates $\mathrm{NO}_{2}, \mathrm{PM}_{2.5}$, and $\mathrm{O}_{3}$ ) [6], and fine particulate matter $\left(\mathrm{PM}_{2.5}\right)$ [7]. In this study, we tested six urban air pollutants and two air quality indices for the relation with emergency department (ED) visits for urticaria.

\section{MATERIAL AND METHODS}

The studied area is the city of Toronto, Canada, well determined by the boundaries of the Census Division (CD) structure. The considered land area is 630.2 square kilometers. In the year 2016, the estimated population of this CD was $2,731,571$ people. The population studied in this work includes males and females of all ages seeking medical care at emergency departments (ED) in Toronto. The National Ambulatory Care Reporting System (NACRS) database, a reporting health system containing diagnosed ED visits, was used as a source of health outcomes. The system has more than $97 \%$ of the ED visits in the province of Ontario. The corresponding health data from the NACRS database were retrieved using SAS Guide 7.1 software (SAS Institute, Cary, NC, USA). The health records have basic patient information including sex, age, date of ED visit, cause of visits (primary), and postal code.

Sex and age were applied to construct separate strata as: all sexes, males, females, and three age groups: 0-10, 11-60, and $60+$ years old. Postal code of patients' residential address 
were used to identify persons in the studied CD. The study period is 4,292 days, from April 1, 2004 to December 31, 2015. Two seasons were defined and considered: warm (April-September) and cold (October-March). The records in the NACRS database were identified by applying ICD-10 code L50 to extract all health cases related to urticaria. Only primary causes of the ED visits were applied to extract the records. The drawn cases were grouped and represented as daily counts.

Six ambient air pollutants were used and their daily average concentration levels were considered as exposure. The potential associations of ED visits for urticaria with the following urban air pollutants were analyzed: fine particulate matter $\left(\mathrm{PM}_{2.5}\right)$, sulphur dioxide $\left(\mathrm{SO}_{2}\right)$, carbon monoxide $(\mathrm{CO})$, nitrogen dioxide $\left(\mathrm{NO}_{2}\right)$, ground-level ozone $\left(\mathrm{O}_{3}-\right.$ as a daily average, $\mathrm{O}_{3}-8$ hour ozone $\left(\mathrm{O}_{3} \mathrm{H} 8\right)$ - as a maximum 8 hour average). In addition, two air quality health indexes (AQHI and AQHI-X) were evaluated and used as a risk factor. The index values encapsulate air pollutants and their associated health risk estimations determined by mortality rates in large Canadian cities. The AQHI values are calculated as follows.

$$
\mathrm{AQHI}=\frac{1000}{10.4} \times\left(\mathrm{e}^{0.000537 * 03}+\mathrm{e}^{0.000871 * \mathrm{NO} 2}+\mathrm{e}^{0.000487 * \mathrm{PM} 2.5}-3\right)
$$

The AQHI-X uses $\mathrm{O}_{3} \mathrm{H} 8$ concentrations [8]. Statistics on six air pollutants and two air quality health indexes is given in [8]. We applied the following statistical model in the R software (version 3.5.1, The R Foundation for Statistical Computing) using the gnm package. The realized statistical models had the following form:

modeFIT $<$ - gnm (EDUtricaria $\sim$ AirPL + ns $($ TempL,3) + ns(RHumL,3), data $=$ dataED, family=quasipoisson, eliminate $=$ factor(Cluster)).
Here air pollutant (AirPL), weather factors (temperature and relative humidity; TempL, RHumL), are lagged (L days) by the same number of days. The Cluster has the form <year:month:day of week $>$. Relative risk is calculated using the coefficient for AirPL.

\section{RESULTS}

Table 1 summarizes the statistics on daily ED visits by 18 considered strata. It also shows the percentages of urticaria (\%All) among all ED visits for skin diseases. The table indicates that ED visits for urticaria are more frequent for females. All contains all ED visits diagnosed as skin problems and identified with the codes from Chapter XII Diseases of the skin and subcutaneous tissue (ICD-10 codes: L00-L99).

All numerical results (from 2,160 models) are presented at: https:/github.com/szyszkowiczm/URTICARIAToronto, in the file ResultL50Toronto.csv. These values are the coefficients (Beta) for air pollutant and their standard error (SE), allowing finding the corresponding relative risks (RR) and the $95 \%$ confidence intervals $(95 \% \mathrm{CI})$. Figure 1 . represents the RRs and their $95 \% \mathrm{CIs}$ for a one IQR increase in concentration of ozone. $\{\mathrm{RR}=\exp ($ Beta*IQR, 95\%CI $=[\exp (($ Beta-1.96*SE)*IQR $)$, $\exp (($ Beta $+1.96 * \mathrm{SE}) * \mathrm{IQR})]\}$.

Figure 2 illustrates all positive statistically significant associations by strata and lags (left panel) and by strata and air pollutants (right panel). It can be seen as a map to the numerical results presented in the file ResultL50Toronto.csv. Statistics on six air pollutants and two air quality health indexes are given in $[8,9]$. In addition, in Supplementary Materials there are presented: map of Toronto, histograms of air pollutants, and histogram of weather factors.

TABLE 1. Statistics on daily ED visits for urticaria (ICD-10 codes: L50). Toronto, Canada, April, 2004 - December, 2015.

\begin{tabular}{|c|c|c|c|c|c|c|c|c|}
\hline Sex/Age/Season & Utricaria & $\%$ All & Min & Q1 & Median & Mean & Q3 & Max \\
\hline All & 36,249 & 11 & 0 & 6 & 8 & 8.4 & 11 & 31 \\
\hline Female & 31,284 & 19 & 0 & 5 & 7 & 7.3 & 9 & 26 \\
\hline Male & 14,927 & 9 & 0 & 2 & 3 & 3.5 & 5 & 14 \\
\hline Warm All & 19,783 & 11 & 0 & 6 & 9 & 9.0 & 11 & 31 \\
\hline Warm Female & 17,176 & 20 & 0 & 5 & 7 & 7.8 & 10 & 26 \\
\hline Warm Male & 8,100 & 9 & 0 & 2 & 3 & 3.7 & 5 & 14 \\
\hline Cold All & 16,466 & 10 & 0 & 6 & 8 & 7.9 & 10 & 21 \\
\hline Cold Female & 14,108 & 17 & 0 & 5 & 6 & 6.7 & 9 & 19 \\
\hline Cold Male & 6,827 & 8 & 0 & 2 & 3 & 3.3 & 4 & 13 \\
\hline Age $0-10$ All & 9,094 & 32 & 0 & 1 & 2 & 2.1 & 3 & 14 \\
\hline Age 0-10 Female & 4,129 & 31 & 0 & 0 & 1 & 1.0 & 2 & 6 \\
\hline Age $0-10$ Male & 4,965 & 32 & 0 & 0 & 1 & 1.2 & 2 & 9 \\
\hline Age 11-60 All & 23,577 & 10 & 0 & 4 & 5 & 5.5 & 7 & 24 \\
\hline Age 11-60 Female & 14,813 & 14 & 0 & 2 & 3 & 3.5 & 5 & 15 \\
\hline Age 11-60 Male & 8,764 & 7 & 0 & 1 & 2 & 2.0 & 3 & 10 \\
\hline Age $60+$ All & 3,578 & 4 & 0 & 0 & 1 & 0.8 & 1 & 6 \\
\hline Age $60+$ Female & 2,380 & 5 & 0 & 0 & 0 & 0.6 & 1 & 5 \\
\hline Age $60+$ Male & 1,198 & 3 & 0 & 0 & 0 & 0.3 & 0 & 4 \\
\hline
\end{tabular}

\%All - percentage of all ED visits for skin problems (L00-L99), Warm: April - September, Cold: October - March, Min - minimum, Max -maximum, Q1-25 $5^{\text {th }}$ percentile, Q3-75 $5^{\text {th }}$ percentile. 


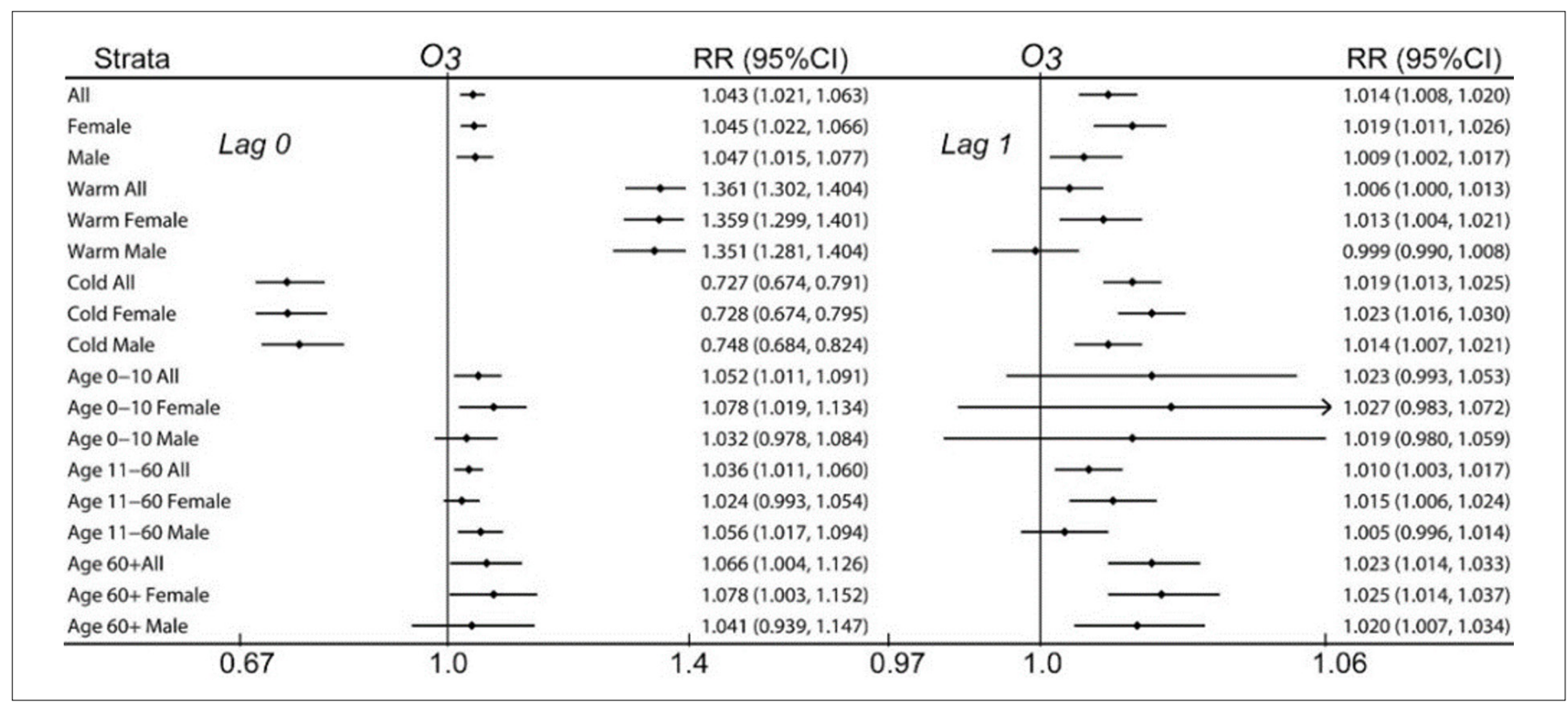

FIGURE 1. Relative risks (RR) and $95 \%$ confidence intervals $(95 \% \mathrm{CI})$ reported for ozone (lag 0 and 1 days). RR and $95 \%$ CIs are shown for a one IQR increase $(\mathrm{IQR}=12.8 \mathrm{ppb})$.

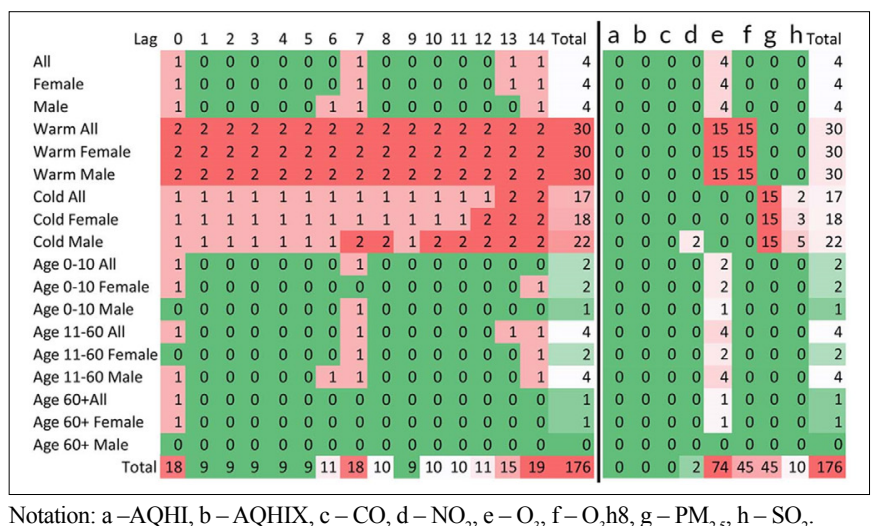

FIGURE 2. Left panel: numbers of positive statistically significant associations by strata and lags, a summary by air pollutants. Right panel: numbers of positive statistically significant associations by strata and air pollutants (a-h), a summary by lags.

\section{DISCUSSION}

The obtained results indicate the strong association between ED visits for urticaria and the concentration of ambient ozone. For lag 0 (same day) exposure the effect is the strongest in the warm period. It is interesting that in the cold period the association changes its sign for lag 0 day (negative) and for lag 1 day (positive).

In total, we obtained 176 statistically significant positive associations. In addition to ambient ozone (both forms), two air pollutants, particulate matter and sulphur dioxide, also show positive associations (45 and 10, respectively). The associations are stronger during the warm period (April-September) and among females. This relationship has been observed previously in other epidemiological work $[6,10-12]$ which also showed increased risk for other skin conditions other than urticaria, and has a biological basis. Laboratory studies [13] have shown that due to ozone's highly oxidizing nature, it can exert oxidative stress on skin cells resulting in an increase of various indicators of inflammation: protein carbonyls (a product of protein oxidation), which in high quantities can inhibit proteasome activity and lead to various diseases depending on the affected location [14], proinflammatory marker cyclooxygenase-2 (COX-2) accompanied by the proliferation of heat shock proteins (HSPs), whose roles in apoptosis, cell proliferation and inflammatory response pathways such as MAPK or NF- $\kappa$ B may result in abnormal skin physiology [15] although their presence is needed to repair misfolded proteins resultant from the inflammatory stress. We also observed that the warm period was significantly more strongly associated to ED visits than the cold period, however this may simply be due to the higher likelihood of skin exposure to ambient air in warmer months. A recent study showed that applying a topical antioxidant may help reduce the effect of ozone-induced oxidation on the skin [16]. Other risk factors such as high population density, farm work, poverty and minority status have all been shown to be associated with an increased risk of developing urticaria [17,18]. While this study shows quite a strong association between air pollution (and in particular ambient ozone) and urticaria, some studies found little or no association between development of urticaria and air pollution [19] and associated the higher rates of urticaria to food allergies and other factors.

There are many limitations in this study. One of them is that we assumed the same levels of exposure for all persons in the study area. The results for all skin conditions and other ICD-10 Chapters (including allergic bronchitis or respiratory issues) are presented at the following location https://github.com/ szyszkowiczm/ResultsToronto. In the location https://github. com/szyszkowiczm/URTICARIAToronto, there is presented a map of the city of Toronto with indicated population density and positions of the seven (nine) air pollution monitors. Histograms of the measured ambient air pollutants and weather factors are also shown. The values of all estimated RRs and their $95 \% \mathrm{CIs}$ for a one IQR increase are reported for all air pollutants, their lags, and strata, i.e. for 2,160 models.

Funding: This research received no external funding.

Institutional Review Board Statement: The Health Canada Research Ethic Board determined that the study is IRB exempt, given that patient data were pre-existing and de-identified.

Informed Consent Statement: Not applicable. 
Data Availability Statement: Health data: NACRS (2020), The National Ambulatory Care Re-porting System, CIHI, Canada; https://www.cihi.ca/en/national-ambulatory-care-reporting-system-metadata. Environmental data: NAPS (2020) Web site: http://maps-cartes.ec.gc.ca/rnspa-naps/data.aspx, Canada.

Acknowledgments: The authors acknowledge Environment Canada for providing the air pollution data from the National Air Pollution Surveillance (NAPS) network.

Parts of this material are based on data and information compiled and provided by the Canadian Institute for Health Information (CIHI). However, the analyses, conclusions, opinions and statements expressed herein are not necessarily those of CIHI.

Conflicts of Interest: The authors declare no conflict of interest. The funders had no role in the design of the study; in the collection, analyses, or interpretation of data; in the writing of the manuscript, or in the decision to publish the results.

\section{REFERENCES}

1. Zuberbier, T. Urticaria. Allergy. 2003;58:1224-34.

2. Kayiran MA, Akdeniz N. Diagnosis and treatment of urticaria in primary care. North Clin Istanb. 2019;6:93-9.

3. Wedi B, Wieczorek D, Raap U, Kapp A. Urticaria. J Dtsch Dermatol Ges. 2014;12:997-1009.

4. Raciborski F, Klak A, Czarnecka-Operacz M, et al. Epidemiology of urticaria in Poland -nationally representative survey results. Post Dermatol Alergol. 2018;35:67-73.

5. Szyszkowicz M, Porada E, Searles G, Rowe B. Ambient ozone and emergency department visits for skin conditions. Air Qual Atmos Health. 2012;5:303-9.

6. Kousha T, Valacchi G. The air quality health index and emergency department visits for urticaria in Windsor, Canada. J Toxicol Environ Health A. 2015;78(8):524-33.

7. Mrad-Nakhlé M, Farah W, Ziade N, et al. Exposure to fine particulate matter and urticaria: an eco-epidemiological time-series analysis in Beirut. Toxicol Environ Health Sci. 2021;13:175-82.

8. Szyszkowicz M, de Angelis N. Ambient air pollution and emergency department visits in Toronto, Canada. Environ Sci Pollut Res Int 2021;28:28789-96.

9. Szyszkowicz M, Schoen S, de Angelis N. Air pollution and Emergency Department visits for Disease of the Genitourinary System. Environ Health Insights. 2021;15:1-10.

10. Xu F, Yan S, Wu M, et al. Ambient ozone pollution as a risk factor for skin disorders. Br J Dermatol. 2011;165(1):224-5.

11. Lefebvre MA, Pham DM, Boussouira B, et al. Evaluation of the impact of urban pollution on the quality of skin: a multicentre study in Mexico. Int $\mathrm{J}$ Cosmet Sci. 2015;37:329-38.

12. Tseng HW, Lu LY, Shiue YL. Short-term impact of ambient air pollution exposure on daily clinic visits for patients with urticaria in Kaohsiung, Taiwan. Air Qual Atmos Health. 2021;14:1063-70.

13. Valacchi G, Van der Vliet A, Schock BC, et al. Ozone exposure activates oxidative stress responses in murine skin. Toxicol. 2002;179:163-70.

14. Dall-Donne I, Aldini G, Carini M, et al. Protein carbonylation, cellular dysfunction, and disease progression. J Cell Mol Med. 2006;10:389-406.

15. Valacchi G, Fortino V, Bocci V. The dual action of ozone on the skin. Br J Dermatol. 2005; 153:1096-100.

16. Pecorelli A, McDaniel DH, Wortzman M, Nelson DB. Protective effects of a comprehensive topical antioxidant against ozone-induced damage in a reconstructed human skin model. Arch Dermatol Res. 2021;313:139-46.

17. JadhavR, Alcala E, Sirota S, Capitman J. Risk factors for acute urticaria in Central California. Int J Environ Res Public Health. 2021;18:3728.

18. He Y, Shi C, Ling F. et al. Short-term effects of outdoor particulate matter pollution on outpatient visits for urticaria in Lanzhou, China. Air Qual Atmos Health. 2021;14:1523-31.

19. Mazur M, Czarnobilska M, Czarnobilska E. Prevalence and potential risk factors of urticaria in the Polish population of children and adolescents. Post Dermatol Alergol. 2020;37:785.

\section{Corresponding author}

Dr hab. Mieszysław Szyszkowicz

Environmental Health Centre: Environmental Health, Science and Research Bureau 50 Columbine Driveway, Ottawa,

ON K1A 0K9 Canada

E-mail: mietek.szyszkowicz@canada.ca; mszyszkowicz@yahoo.ca 